\title{
The complexity of occupational stress electroencephalogram
}

\author{
Honger Tian ${ }^{1}$, Lili Cao ${ }^{1}$, Jun Wang ${ }^{2}$, Tian $\mathrm{Xu}^{1}$, Yongguo Zhan ${ }^{1}$, Ling Liu ${ }^{1}$ \\ ${ }^{1}$ Key Laboratory of Environment Medicine and Engineering, Ministry of Education, School of Public Health, Southeast University, \\ Nanjing, China \\ ${ }^{2}$ Image Processing and Image Communications Key Lab, College of Geo \& Bio Information, Nanjing University of Posts \& Tele- \\ comm, Nanjing, China; ${ }^{*}$ Corresponding Author: tianhonger0@163.com
}

Received 18 September 2013; revised 20 October 2013; accepted 4 November 2013

Copyright (C) 2013 Honger Tian et al. This is an open access article distributed under the Creative Commons Attribution License, which permits unrestricted use, distribution, and reproduction in any medium, provided the original work is properly cited. In accordance of the Creative Commons Attribution License all Copyrights (C) 2013 are reserved for SCIRP and the owner of the intellectual property Honger Tian et al. All Copyright (C) 2013 are guarded by law and by SCIRP as a guardian.

\begin{abstract}
It is an important method for using electroencephalogram (EEG) to detect and diagnose occupational Stress in clinical practice. In this paper, the complexity analysis method based on Jensen-Shannon Divergence was used to calculate the complexity of occupational stress electroencephalogram from students and nurses. The study found that the complexity of nurses' EEG was higher than that of students' EEG. The result can be used to assisted clinical diagnosis.
\end{abstract}

Keywords: Occupational Stress;

Electroencephalogram; Students; Nurses; Jensen-Shannon Divergence

\section{INTRODUCTION}

Since Jensen-Shannon Divergence (JSD) [1] (which was used to measure the difference between the probability distribution of random variables) was proposed in 1991, it was widely applied to the symbol sequence analysis and characterization [2], such as pattern recognition [3], DNA sequence segmentation. JSD is the result of symmetrizing and smoothing the Kullback-Leibler Divergence (KLD). The non-negativity, symmetry, continuity $[4,5]$ and boundness features of JSD have been widely used in the analysis of time series. Electroencephalogram (EEG) can also be seen as a time series, so we consider using JSD complexity based analysis method to achieve recognition and detection of EEG. In this paper, 12 graduate students from Southeast University and 12 nurses from a third-grade class-A hospital were chosen for comparative analysis.
Nurses need to withstand pressure from work, patient and family. The occupational stress can cause changes in psychology, physiology and behavior, such as anxiety, irritability, depression, chronic fatigue syndrome, sleep disorders, immune system suppression, cardiovascular system disease, aggression, and bad habits [6-9]. Graduate students in universities relatively have lower occupational stress than the nurses. So their EEG should have a different dynamic complexity. The Jensen-Shannon Divergence was used to quantitatively analyze time series. As a statistic parameter in information statistics, JensenShannon Divergence described the complexity between different signals and the entropy value increased with increased complexity.

\section{JENSEN-SHANNON DIVERGENCE (JSD)}

Proposed that $p_{1}, p_{2}$ were two probability distribution of discrete random variables $X$. KULLBACK defined direct difference $I$ as:

$$
I\left(p_{1}, p_{2}\right)=\sum_{x \in X} p_{1}(x) \log \frac{p_{1}(x)}{p_{2}(x)}
$$

It can be seen from Equation (1) that differences $I$ has non-negativity and incremental feature, but it does not have symmetry. In order to meet this point, the form of a symmetric difference- $\mathrm{J}$ difference was proposed:

$$
\begin{aligned}
& J\left(p_{1}, p_{2}\right) \\
& =I\left(p_{1}, p_{2}\right)+I\left(p_{2}, p_{1}\right) \\
& =\sum_{x \in X}\left(p_{1}(x)-p_{2}(x)\right) \log \frac{p_{1}(x)}{p_{2}(x)}
\end{aligned}
$$

Distance change in the of two probability distributions can meet the metric nature. The distance change was 
defined as follows:

$$
V\left(p_{1}, p_{2}\right)=\sum_{x \in X}\left|p_{1}(x)-p_{2}(x)\right|
$$

For the relationship between the direct differences $I$ and distance changes $V$, it was found the minimum value of the differences $I$ based on the $V$ :

$$
I\left(p_{1}, p_{2}\right) \geq \max \left\{L_{1}\left(V\left(p_{1}, p_{2}\right)\right), L_{2}\left(V\left(p_{1}, p_{2}\right)\right)\right\}
$$

where,

$$
\begin{aligned}
& L_{1}\left(V\left(p_{1}, p_{2}\right)\right) \\
& =\log \frac{2+V\left(p_{1}, p_{2}\right)}{2-V\left(p_{1}, p_{2}\right)}+\frac{2 V\left(p_{1}, p_{2}\right)}{2+V\left(p_{1}, p_{2}\right)}, 0 \leq V\left(p_{1}, p_{2}\right) \leq 2 \\
& L_{2}\left(V\left(p_{1}, p_{2}\right)\right)=\frac{V^{2}\left(p_{1}, p_{2}\right)}{2}+\frac{V^{4}\left(p_{1}, p_{2}\right)}{36} \\
& +\frac{V^{6}\left(p_{1}, p_{2}\right)}{288}, 0 \leq V\left(p_{1}, p_{2}\right) \leq 2
\end{aligned}
$$

But there are not a general representation of the maximum value for direct differences $I$ and differences $J$ based on $V$. So there exists some limitations when measuring the difference between the probability distribution.

For generally descripting the maximum limit of the difference, papers [1,9] gave the amended definition of $I$ and $J$ :

$$
\begin{aligned}
K\left(p_{1}, p_{2}\right) & =\sum_{x \in X} p_{1}(x) \log \frac{p_{1}(x)}{\frac{1}{2} p_{1}(x)+\frac{1}{2} p_{2}(x)} \\
& =I\left(p_{1}, \frac{1}{2} p_{1}+\frac{1}{2} p_{2}\right)
\end{aligned}
$$

The corresponding symmetrical form difference was as follows:

$$
L\left(p_{1}, p_{2}\right)=K\left(p_{1}, p_{2}\right)+K\left(p_{2}, p_{1}\right)
$$

It can be seen from Equation (8) that, the difference between $K$ and $L$ was not only meeting the non-negativity but also having limitation and semi-bounded. That is:

$$
\begin{aligned}
& K\left(p_{1}, p_{2}\right)<+\infty, K\left(p_{1}, p_{2}\right) \geq K\left(p_{1}, p_{1}\right) \\
& L\left(p_{1}, p_{2}\right)<+\infty, L\left(p_{1}, p_{2}\right) \geq L\left(p_{1}, p_{1}\right) \\
& L\left(p_{1}, p_{2}\right)=2 H\left(\frac{p_{1}+p_{2}}{2}\right)-H\left(p_{1}\right)-H\left(p_{2}\right)
\end{aligned}
$$

Now setting $\pi_{1}$ and $\pi_{2}$ were the weight of two probability distributions, and meeting

$\pi_{1}, \pi_{2} \geq 0, \pi_{1}+\pi_{2}=1$, Jensen-Shannon Divergence (JSD) was defined as:

$$
J S_{\pi}\left(p_{1}, p_{2}\right)=H\left(\pi_{1} p_{1}+\pi_{2} p_{2}\right)-\pi_{1} H\left(p_{1}\right)-\pi_{2} H\left(p_{2}\right)
$$

where $H(P)=\sum_{j=1}^{N} p_{j} \ln \left(p_{j}\right)$ is Shannon Entropy.

For more than two but a limited number of probability distributions $p_{1}, p_{2}, \cdots, p_{n}$, the corresponding weight were $\pi_{1}, \pi_{2}, \ldots, \pi_{n}$ respectively, JSD was defined as:

$$
J S_{\pi}\left(p_{1}, p_{2}, \cdots, p_{n}\right)=H\left(\sum_{i=1}^{n} \pi_{i} p_{i}\right)-\sum_{i=1}^{n} \pi_{i} H\left(p_{i}\right)
$$

\section{DATA ANALYSIS}

The EEG data we used were taken from 12 students and 12 nurses. The sampling frequency was $200 \mathrm{~Hz}$. We used JSD to analyze the complexity of the EEG data of 12 students and 12 nurses and the corresponding results were shown in Table 1.

According to Table 1, we can plot the complexity measures of three ECG signals and twelve were shown in Figure 1.

It can be seen from the Figure 1 that, the JensenShannon Divergence value of students is less than that of nurses. The $\mathrm{T}$ test value of the two groups was equal to 4.414 which confidence probability is 0.001 . It indicated that high level of occupational stress had a higher JensenShannon Divergence value than low level, and EEG should be more complex. So students and nurses can be statistically distinguished.

\section{CONCLUSION}

In this paper, the complexity analysis method based on Jensen-Shannon Divergence was used to calculate the complexity of occupational stress electroencephalogram

Table 1. Complexity measures of twelve ECG signals (2000 points).

\begin{tabular}{ccc}
\hline Subjects & Student & Nurse \\
\hline 1 & 0.2163 & 0.2215 \\
2 & 0.2175 & 0.2787 \\
3 & 0.0599 & 0.2813 \\
4 & 0.2092 & 0.2403 \\
5 & 0.1929 & 0.2659 \\
6 & 0.0694 & 0.2793 \\
7 & 0.2171 & 0.2727 \\
8 & 0.2358 & 0.2809 \\
9 & 0.0692 & 0.2714 \\
10 & 0.2145 & 0.2707 \\
11 & 0.2055 & 0.2798 \\
12 & 0.0621 & 0.2743 \\
mean \pm STD & $0.1641 \pm 0.0738$ & $0.2681 \pm 0.0184$ \\
\hline
\end{tabular}




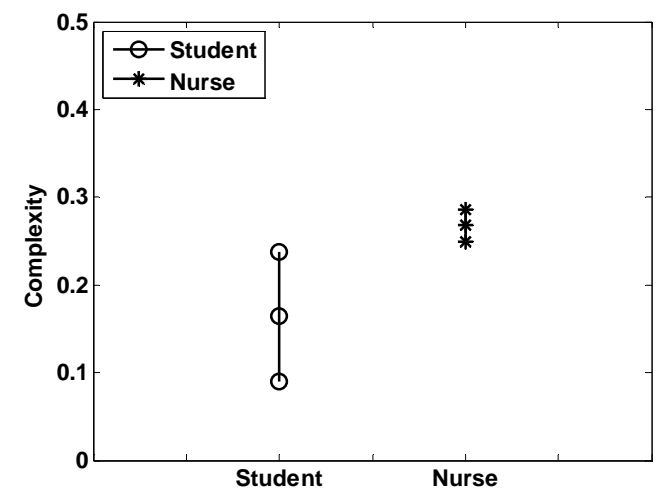

Figure 1. Dynamic range of two kind signals' complexity $(\mathrm{N}=2000)($ mean $\pm \mathrm{STD}) . \circ($ Student $)$, * (Nurse).

from students and nurses. The study found that the complexity of nurses' EEG was higher than that of students' EEG. The result can be used to assisted clinical diagnosis.

\section{ACKNOWLEDGEMENTS}

This work was supported by the National Natural Science Foundation of China (Grant Nos. 61271082, 61201029, 61102094), the Natural Science Foundation of Jiangsu Province (Grant Nos. BK2011759, BK2011565), the Social development Foundation-science and technology support projects of Jiangsu province (Grant Nos. BE2011777), and Foundation of Nanjing University of Posts and Telecommunications (JG03212JX02, JG03210JX19, 2011XSG11).

\section{REFERENCES}

[1] Lin, J.H. (1991) Divergence measure based on the Shannon entropy. IEEE Transactions on Information Theory, 37, 145-151. http://dx.doi.org/10.1109/18.61115
[2] Lamberti, P.W. and Majtey, A.P. (2003) Non-logarithmic Jensen-Shannon divergence. Physica A: Statistical Mechanics and Its Applications, 329, 81-90. http://dx.doi.org/10.1016/S0378-4371(03)00566-1

[3] Hung, W.L. and Yang, M.S. (2008) On the J-divergence of intuitionistic fuzzy sets with its application to pattern recognition. Information Sciences, 178, 1641-1650. http://dx.doi.org/10.1016/j.ins.2007.11.006

[4] Rosso, O.A., Larrondo, H.A., Martin, M.T. and Fuentes, M.A. (2007) Distinguishing Noise from Chaos. Physical review letters, 99, Article ID: 154102. http://dx.doi.org/10.1103/PhysRevLett.99.154102

[5] Martin, M.T., Plastino, A. and Rosso, O.A. (2006) Generalized statistical complexity measures: Geometrical and analytical properties. Physica A: Statistical Mechanics and Its Applications, 369, 439-462. http://dx.doi.org/10.1016/j.physa.2005.11.053

[6] Bagaajav, A., Myagmarjav, S., Nanjid, K., Otgon, S. and Chae, Y.M. (2011) Burnout and job stress among Mongolian doctors and nurses. Industrial Health, 49, 582-588. http://dx.doi.org/10.2486/indhealth.MS1256

[7] Kikuchi, Y., Nakaya, M., Ikeda, M., Narita, K., Takeda, M. and Nishi, M. (2010) Effort-reward imbalance and depressive state in nurses. Occupational Medicine, 60, 231-233. http://dx.doi.org/10.1093/occmed/kqp167

[8] Voltmer, E., Wingenfeld, K., Spahn, C., Driessen, M. and Schulz, M. (2012) Work-related behaviour and experience patterns of nurses in different professional stages and settings compared to physicians in Germany. International Journal of Mental Health Nursing, 22, 180-189. http://dx.doi.org/10.1111/j.1447-0349.2012.00855.x

[9] Kikuchi, Y., Nakaya, M., Ikeda, M., Takeda, M. and Nishi, M. (2013) Job stress and temperaments in female nurses. Occupational Medicine, 63, 123-128. http://dx.doi.org/10.1093/occmed/kqs212 